\title{
Estado de Salud Bucal en Adolescentes de la Ciudad de México
}

\author{
Oral health status of adolescents in México City
}

\author{
Miriam Ortega-Maldonado, Vanesa Mota-Sanhua y Juan C. López-Vivanco \\ Clínica ABC Amistad. Centro Médico ABC, Santa Fe. México. mortegam@abchospital.com; \\ vmotas@abchospital.com; jclopezv@abchospital.com \\ Recibido 12 Diciembre 2006/Enviado para Modificación14 de Julio 2007/Aceptado 2 Agosto 2007
}

\section{RESUMEN}

Objetivo Determinar el estado de salud bucal (caries dental y gingivitis) en adolescentes de la Ciudad de México, así como el patrón de afección por tipo de diente y las necesidades de tratamiento dental.

Material y Métodos Se realizó un estudio transversal en 590 escolares, entre 13 y 16 años de edad. La exploración bucal formó parte del diagnóstico integral del Programa Escuelas Promotoras de Salud. La presencia de caries dental se definió a partir del índice CPOD (diente cariado, perdido y obturado). La presencia de gingivitis se determinó por el diagnóstico sin sonda propuesto por el CONAVE. Se aplicaron pruebas de $\mathrm{X}^{2}$ y se calcularon riesgos para evaluar la relación de caries, gingivitis y patrón de afección por el tipo de diente acorde con edad y sexo.

Resultados La prevalencia de caries dental fue del 92,2 \%, encontrando un índice CPOD de 7,3; la prevalencia de gingivitis fue de 13,7 \%. La edad de 14 o más años representó un riesgo estadísticamente significativo de caries dental (RMP $=3,1$; IC95: 1,5 - 6,4). El índice de necesidades de tratamiento para caries fue del 95,7 \%, lo que representa un gasto de 642450 pesos mexicanos (59 818,4 dólares americanos).

Discusión Con relación al estado de salud bucal en adolescentes, la caries dental es la enfermedad de mayor prevalencia y su riesgo aumenta con la edad. En promedio, el índice CPOD fue dos veces mayor que los estándares establecidos por la Organización Mundial de la Salud-OMS.

Palabras Clave: Salud bucal, caries dental, adolescente (fuente: DeCS, BIREME).

\section{ABSTRACT}

Objective Determining the oral health status (dental decay and gingivitis) of adolescents from Mexico City and affection pattern by tooth type and treatment needs. Material and Methods A cross-sectional study was designed for 590 adolescents aged 13 to 16 from an urban area of Mexico City. Oral exploration formed part of the health diagnosis provided by the Schools Promoting Health Programme. The DMFT (decayed, missing and filled teeth) index was used for determining dental decay. Diagnosis with no probe was used for evaluating gingivitis. Chi2 tests were applied 
and risks were calculated for evaluating the association between dental decay, gingivitis and affection pattern by tooth type according to sex and age.

Results The prevalence of dental decay and gingivitis was $92,2 \%$ and $13,7 \%$, respectively. DMFT index was 7,3 . Being aged 14 and over represented a significant risk for dental decay $(\mathrm{OR}=3,1 ; \mathrm{Cl} 95 \%=1,5-6,4)$. The treatment needs index for dental decay was $95,7 \%$, representing an expenditure of 642450 Mexican pesos (59 818,4 US dollars).

Discussion Regarding adolescents' oral health status, dental caries was the affection having the highest prevalence and its risk increased with age. The DMFT index was twice as high as WHO standards.

Key Words: Oral health, dental caries, adolescent (source: MeSH, NLM).

$\mathrm{E}$ l adecuado estado de la salud bucal permite mantener funciones vitales como la alimentación, la comunicación y el afecto (1). Un indicador de la salud bucal es el índice de caries dental (sumatoria de número de dientes cariados, perdidos y obturados: CPOD). Este índice, muestra problemas no resueltos, presentes y futuros $(2,3)$, además de ser ampliamente utilizado en estudios epidemiológicos para estimar la magnitud de problemas de salud bucal.

En 1980, la Secretaría de Salud (SS), realizó una encuesta de morbilidad bucal en escolares del Distrito Federal, cuyos datos indicaron que el nivel de caries había aumentado con respecto a la meta establecida por la Organización Mundial de la Salud (4). Asimismo, Irigoyen en 1988 realizó un estudio en los estados de Baja California, Tabasco, Yucatán y el Estado de México, el cual se utilizó como línea basal para determinar la prevalencia de caries en los escolares del país cuyo promedio de edad oscilaba entre los 12 años. En este reporte, el índice CPOD se estimo entre 4,5 y 6,5 (5).

Los datos de estudios realizados en escolares en 1997 acerca de la demanda de atención odontológica en los servicios de salud en México indicaron que la caries se trata de un padecimiento que afecta alrededor del $70 \%$ en escolares de secundaria y que ocasiona ausentismo escolar, dolor y pérdida prematura de dientes. Lo cual ocasiona después de los 15 años el 38\% de la pérdida dental $(6,7)$.

Otro problema, considerado de importancia para la salud bucal, son las enfermedades periodontales tales como la gingivitis y la periodontitis. 
Actualmente se conoce que se presentan con mayor prevalencia y severidad en hombres antes de los 20 años (8-10). Esto hace relevante conocer la magnitud del problema en etapas tempranas de la vida, con la finalidad de evitar pérdidas dentales en el futuro.

El propósito del estudio fue determinar de manera observacional el estado de la salud bucal (caries dental y gingivitis) en adolescentes entre 13 y 16 años de edad de la Ciudad de México. Adicionalmente, se evaluó el patrón de afección por tipo de diente y las necesidades de tratamiento dental. En México, se conoce poco sobre estimados de necesidades de tratamiento. La identificación de los problemas prioritarios en el estado de salud bucal y la estimación de las necesidades de su tratamiento, darán pautas para establecer programas de salud bucodental contextualizados hacia la población estudiada.

\section{MATERIAL Y MÉTODOS}

La Clínica ABC Amistad del Centro Médico ABC, cuya misión es brindar atención primaria a comunidades marginadas, en diciembre del 2005 realizó un censo en la Colonia de Corpus Cristi, de la delegación Álvaro Obregón, Ciudad de México. En dicho censo se identificaron las escuelas públicas susceptibles de integrarse al Programa de Escuelas Promotoras de Salud que imparte la clínica (descrito por la OPS que contempla valoración e intervenciones médica y bucodental en escolares) (11). De tal manera, la escuela secundaria pública registrada en el censo quedó integrada al programa.

El Programa de Escuelas Promotoras de Salud, cuenta con la aprobación del Comité de Beneficencia del Centro Médico ABC y la autorización de la Secretaría de Educación Pública. El presente estudio forma parte de este programa.

El diseño del estudio fue transversal analítico. El universo consistió en 701 escolares. De ellos, 54, 7\% esto es, 322 eran mujeres. La muestra de estudio la integraron 590 adolescentes que representó el 84\% de la población universo. La muestra de estudio quedó constituida por aquellos que cumplieron con los siguientes criterios de selección: a) adolescentes de ambos sexos, b) con edades entre 13 y 16 años, c) asistir en forma voluntaria a la valoración bucodental de la Clínica de Beneficencia durante enero de 2006 y e) con el consentimiento informado de sus padres, a quienes también se les informó que la atención de los problemas de salud bucal de sus hijos serían abordados por el Equipo de Atención Primaria en la Clínica de Beneficencia. 
El examen odontológico se realizó por un evaluador previamente calibrado y consistió en la valoración de caries, gingivitis. La valoración de caries dental incluyó la determinación de dientes cariados, perdidos y obturados en dentición permanente. A partir de esta información se calculó el índice CPOD y se determinó el patrón de afectación por tipo de diente. Para la determinación de la gingivitis se utilizó el diagnóstico sin sonda propuesto por el CONAVE, el cual estima el estado periodontal de la boca a partir de la presencia de inflamación y sangrado. Los datos se recabaron en historias clínicas bucodentales. Cabe mencionar, que durante el estudio se involucran mediciones de rutina que no implican riesgo para el adolescente.

Las necesidades de tratamiento se evaluaron a partir de la razón del número de dientes cariados entre el valor obtenido del índice CPOD. El costo de atención se calculó a partir del costo de la rehabilitación por diente cariado 150 pesos mexicanos (14,01 dólares americanos) y por diente perdido 200 pesos mexicanos (18,69 dólares americanos).

Se elaboró una base de datos en el paquete SPSS (Statistical Pacgage for the Social Sciences ${ }^{\mathrm{TM}}$, versión 13.0), para el mantenimiento de la calidad de los datos y el análisis de los mismos. Se obtuvieron frecuencias y porcentajes para las variables cualitativas sexo, presencia de gingivitis, caries y medidas de tendencia central y de dispersión para la variable edad, número de dientes con caries e índice CPOD. Se aplicaron pruebas de $\mathrm{x}^{2}$ para evaluar la relación entre el índice COPD y gingivitis por sexo y edad. Así mismo, se evaluó la relación entre el patrón de afección por tipo de diente y la edad.

Se calcularon razones de momios para la prevalencia de caries dental y gingivitis por edad y sexo. Así como, del patrón de afección por tipo de diente y edad.

\section{RESULTADOS}

En total, se evaluaron 590 adolescentes. De ellos, 54,7 \%, es decir, 322 fueron mujeres. La mediana de edad fue de 13 años, siendo 12 años la edad mínima y 16 la máxima. La prevalencia de caries en los adolescentes fue de 92,2 \% y de gingivitis de 13,9\%. El promedio del índice CPOD fue 7,32 (D.E. + 4,98).

En la Tabla 1, se encuentran las frecuencias y riesgos de caries de acuerdo con la edad y el sexo. Los adolescentes de $\geq 14$ años de edad tienen mayor 
riesgo de presentar caries que los adolescentes $\leq 13$ años. Este riesgo fue estadísticamente significativo.

En la Tabla 2, se presentan las frecuencias y riesgos de gingivitis por edad y sexo. Como se puede ver, el grupo de edad de $\geq 14$ años tiene más riesgo de presentar gingivitis en comparación con el grupo de $\leq 13$ años. Además, los hombres tuvieron mayor riesgo de gingivitis. Estos riesgos fueron estadísticamente significativo.

Tabla 1. Riesgos de caries de acuerdo con la edad y el sexo de los adolescentes

\begin{tabular}{|c|c|c|c|c|c|c|c|}
\hline \multirow{3}{*}{$\begin{array}{l}\text { Edad } \\
\text { (años) }\end{array}$} & \multicolumn{4}{|c|}{ Caries dental } & \multirow[b]{3}{*}{$\mathrm{p} \dagger$} & \multirow[b]{3}{*}{ RM } & \multirow[b]{3}{*}{ IC al $95 \%$} \\
\hline & \multicolumn{2}{|c|}{$\begin{array}{l}\text { Presente } \\
(n=544)\end{array}$} & \multicolumn{2}{|c|}{$\begin{array}{l}\text { Ausente } \\
(n=46)\end{array}$} & & & \\
\hline & $\mathrm{n}$ & $\%$ & n & $\%$ & & & \\
\hline$\leq 13$ & 292 & 53,7 & 36 & 78,3 & $0,002 \uparrow$ & 3,1 & $(1,5-6,7)$ \\
\hline$\geq 14$ & 252 & 46,3 & 10 & 21,7 & & & \\
\hline \multicolumn{8}{|l|}{ Sexo } \\
\hline Masculino & 249 & 42,2 & 22 & 47,9 & 0,908 & 0,9 & $(0,5-1,7)$ \\
\hline Femenino & 295 & 50,0 & 24 & 52,2 & & & \\
\hline
\end{tabular}

Tabla 2. Riesgos de gingivitis de acuerdo con la edad y el sexo de los adolescentes

\begin{tabular}{|c|c|c|c|c|c|c|c|}
\hline \multirow{3}{*}{$\begin{array}{l}\text { Edad } \\
\text { (años) }\end{array}$} & \multicolumn{4}{|c|}{ Gingivitis } & \multirow[b]{3}{*}{ p† } & \multirow[b]{3}{*}{ RM } & \multirow[b]{3}{*}{ IC al $95 \%$} \\
\hline & \multicolumn{2}{|c|}{$\begin{array}{l}\text { Presente } \\
(n=81)\end{array}$} & \multicolumn{2}{|c|}{$\begin{array}{l}\text { Ausente } \\
(n=509)\end{array}$} & & & \\
\hline & $n$ & $\%$ & $n$ & $\%$ & & & \\
\hline$\leq 13$ & 45 & 12,3 & 217 & 20,8 & $0 ; 039 ;$ & 1,7 & $(1,0-2,7)$ \\
\hline$\geq 14$ & 36 & 60,5 & 292 & 67,4 & & & \\
\hline Sexo & & & & & & & \\
\hline Masculino & 46 & 56,8 & 225 & 44.2 & $0.046 \mp$ & 1.658 & $(1,033-2,662)$ \\
\hline Femenino & 35 & 43,2 & 284 & 55.8 & & & \\
\hline
\end{tabular}

¡ valor de p estadisticamente significativo; *RM estadisticamente significativa

Con relación a la presencia de caries dental por el patrón de afectación dependiendo el tipo de diente, se encontró que los molares y los premolares son las dientes más afectados, seguidos por los caninos y los incisivos. La frecuencia de la enfermedad fue mayor en los del maxilar que en los de la mandíbula.

$\mathrm{Al}$ analizar el problema de caries por tipo de diente y edad; se observa que, con relación al maxilar, los adolescentes de $\geq 14$ años tienen más riesgo de presentar caries en molares que los de $\leq 13$ años (RM=3,6; IC95 \%: 2,1-6,2). Asi mismo, los escolares de $\geq 14$ años presentaron más riesgo de caries en los premolares en comparación con los escolares de $\leq 13$ años (RM=5; IC95 \%: $3,0-8,1)$. Respecto a la presencia de caries en los molares y premolares de la 
mandíbula, los adolescentes de $\geq 14$ años tienen 4 veces más riesgo que los de menor edad (RM= 4,4; IC95 \%: 2,7 - 7,5 y RM= 4,2; IC95 \%: 2,3-7,8 respectivamente). Lo cual puede explicarse al periodo de erupción de los mismos.

El índice de necesidades de tratamiento dental para caries fue del 9,7 \%, mientras que para el problema de pérdida dental fue del 0,2\%.

A partir de los resultados del índice CPOD, se calculó el costo de ltratamiento. En los adolescentes evaluados, se identificaron 4283 piezas cariadas. Considerando que el costo de atención por diente es de 150,00 pesos mexicanos (14,01 dólares americanos), el costo total resulto ser de 642450 pesos mexicanos (59 818, 4 dólares).

Con relación a la pérdida dental, el total de dientes perdidos fue de 10 . Considerando que el costo de atención, por cada reemplazo es de 200 pesos mexicanos (18,69 dólares americanos), el costo para atender la rehabilitación dental, resulto ser de 2000 pesos mexicanos (186, 91 dólares).

\section{DISCUSIÓN}

La OMS y la Federación Dental Internacional, propusieron como meta para el año 2020, que en niños menores de 12 años existiera una prevalencia de caries dental en tres dientes como máximo $(4,12)$.

Los datos obtenidos y analizados en el presente estudio, indican que enlos adolescentes, el 92, 2 \% presenta caries dental. El índice CPOD encontrado fue de 7. Estos hallazgos, reflejan una brecha de más del doble respecto a la meta anteriormente mencionada. Además, el porcentaje de necesidades de tratamiento dental observado fue elevado.

El número de dientes afectados se ubicó en un intervalo de 0 a 10 . Lo cual coincide con los intervalos reportados en Cuba, en donde se observa que a pesar de la disminución en la prevalencia de caries dental, el intervalo de dientes afectados se mantiene (13).

Respecto al análisis de caries dental por sexo, se observó que las mujeres fueron las más afectadas, sin embargo, presentaron en mayor número dientes tratados (28,21 \%) y menor porcentaje de afección por gingivitis (10,97 \% ). Este mismo comportamiento se ha visto en población adulta (9). 
Es probable que la mayor prevalencia de daños en las mujeres, se deba al inicio temprano de la erupción dental. Esto origina que las mujeres se encuentren expuestas a más temprana edad a factores cariogénicos y a necesidades de tratamiento (14).

Cabe señalar que al analizar los resultados del estudio, se observa que por cada pérdida dental hay 108,8 dientes cariados y 7 escolares con presencia de gingivitis. Con estos datos y de acuerdo con lo referido por la OMS y la Norma Oficial Mexicana, la caries dental mantiene su tendencia y ocupa el principal problema de morbilidad bucal de la población escolar $(4,15)$. Por esta razón, los programas de educación para salud bucal del país, han sido dirigidos hacia el problema de caries dental.

En México, no se conocen estudios en los que se estimen las necesidades de tratamiento a partir de la descripción epidemiológica de los problemas en la salud bucal. Con los hallazgos obtenidos en este estudio, se observan las necesidades de tratamiento de una población específica. Los resultados presentados, justifican la importancia de realizar más estudios al respecto para que, con base en ellos, se desarrollen programas que atiendan necesidades representativas de la población a la que vayan dirigidos.

Es importante aclarar que la muestra estuvo conformada por escolares de una sola escuela, por lo que los resultados obtenidos sólo pueden extrapolarse en escuelas con características similares

Agradecimiento. El financiamiento del estudio lo proporcionóla Clínica ABC Amistad, Centro Médico ABC.

\section{REFERENCIAS}

1. Petersen E. The world oral health report 2003: continuous improvement or oral health in the 21st century. Rev. Community Dentistry and Oral Epidemiology 2003; 31 (suppl 1): 3-23.

2. OMS. Métodos y programas de prevención de las enfermedades buco-dentales. Serie de Informes Técnicos 713. Ginebra 1984;11-12

3. Irigoyen ME, Zepeda MA, Sánchez L, Molina N. Prevalencia e incidencia de caries dental y hábitos de higiene bucal en un grupo de escolares del sur de la Ciudad de México: Estudio de seguimiento longitudinal. Rev. Asociación Dental Mexicana2001; 53(3):98-104. 
4. Hobdell M, Petersen PE, Clarkson J. Goals for oral health 2020. International Dental Journal 2003; 53:285-288.

5. Moreno GA, Carreón J, Alvear G, López S, Vega L. Riesgo de caries en escolares de escuelas oficiales de la ciudad de México. Revmex Pediatr 2001;68(6):228-233

6. Ralph E, MacdonaldDR. Odontología Pediátrica y del adolescente. 5a Edición. Argentina, Editorial Panamericana; 2002.

7. Nava J, Padilla M, Becerril C. Estado de salud dental y necesidades de tratamiento en escolares entre 6 y 12 años. Estudio en una población de San Mateo Atenco, Edo. de México. Práctica Odontológica 2001; 2 (4): 24-29.

8. Jensen K, Hermosillo GG. Oral health: Dental Caries; Oral hygiene and gingivitis in a deprived urban population in México. Boletín de la oficina Sanitaria Panamericana 1983; 94(6): 587-602

9. Seif TR. Cariología. Prevención, Diagnóstico y Tratamiento Contemporáneo de la Caries Dental. Venezuela. Editorial Actualidades Médico Odontológicas Latinoamérica; 1997.

10. Hernández JR, TelloT, Hernández FJ, Rosette R. Enfermedad periodontal: prevalencia y algunos factores asociados en escolares de una región mexicana. Rev. Asociación Dental Mexicana 2000; 52(6): 222-230

11. OPS/OMS. Escuelas Promotoras de Salud: fortalecimiento de la Iniciativa Regional, Serie Promoción de la Salud No. 14. Washington D.C., 2003.

12. Informe Conjunto de Trabajo de Salud Oral. Secretaría de Salud de México. [Organización Panamericana de la Salud]. 1998; 2:6

13. Delgado ML, Rodíguez CA, Sosa RM, FelipeAA. Estado de salud bucal en la población cubana,1995. Rev Cubana de Estomatología 1999; 37(3):217-29.

14. Madaleno M, Munist MM, Serrano CV, Silver TJ. Sexualidad y adolescencia. La personalidad en el contexto de la salud integral de los adolescentes. En: La salud del adolescente y el joven. Washington, DC: Organización Panamericana dela Salud - Organización Mundial de la Salud; 1994: 1-7.

15. Modificación a la Norma Oficial Mexicana NOM-013-SSA2-1994, Para la prevención y control de enfermedades bucales, publicada el 6 de enero de 1995. 